\title{
Prolonged renal failure in the course of atypical ethylene glycol intoxication
}

\author{
Tomasz Liberek ${ }^{\circledR}$, Julita Śliwarska², Krzysztof Czurak¹, Agnieszka Perkowska-Ptasińska³, \\ Ewa Weber ${ }^{1}$ and Bolesław Rutkowski ${ }^{1}$
}

'Department of Nephrology, Transplantology and Internal Medicine, Gdansk Medical University, Gdańsk, Poland; ${ }^{2}$ Diaverum Dialysis Unit in Starogard Gdański, Starogard Gdański, Poland; ${ }^{3}$ Department of Transplantation Medicine and Nephrology, Transplantation Institute, Warsaw Medical University, Warsaw, Poland

\begin{abstract}
Ethylene glycol poisoning is not an uncommon cause of an acute renal injury. In this paper we present case of prolonged renal failure in the course of ethylene glycol intoxication. Due to the low dose of ingested ethylene glycol and concomitant ethanol consumption, the neurodepressive phase of the intoxication was rather mild and patient presented to the hospital on the ninth day after poisoning with established renal failure. The diagnosis of the specific cause of renal injury was facilitated by the renal biopsy.
\end{abstract}

Key words: ethylene glycol, intoxication, renal failure

Received: 07 October, 2013; revised: 12 November, 2013; accepted: 13 November, 2013; available on-line: 22 November, 2013

\section{INTRODUCTION}

Ethylene glycol ingestion is not an uncommon cause of an acute renal injury. As other types of toxic alcohol poisoning it usually presents in a setting of a progressive loss of consciousness leading to stupor or even coma. These symptoms may be preceded by nausea, vomiting, and abdominal pain. Due to accumulation of acidic metabolites (glycolic, glioxalic and oxalic acid) nonrespiratory acidosis develops with markedly increased anion gap. Plasma osmolar gap (difference between measured and calculated plasma osmolality) can be detected, due to the presence of ethylene glycol itself and its metabolites (Kraut \& Kurtz, 2008). As ethylene glycol is finally metabolized to oxalic acid, it forms very characteristic calcium oxalate monohydrate crystals (known as "whewellite"), which can be detected in urine by microscopic examination and help with diagnosis (Reddy et al., 2006). Poisoning can by confirmed by measuring ethylene glycol levels in plasma which is best performed by gas chromatography, although it is not readily available in many hospitals. Ethylene glycol poisoning typically results in an acute kidney injury leading to acute tubular necrosis and kidney failure usually necessitating dialysis. The mechanism of kidney injury has not been fully elucidated, however, direct toxicity of metabolites and deposition of calcium oxalate has been implicated in this process. The treatment of acute ethylene glycol poisoning, apart from supportive therapy, is focused on slowing down ethylene glycol metabolism to more toxic products by blocking alcohol dehydrogenase, enzyme responsible for metabolizing ethylene glycol to glucoaldehyde. This can be achieved by administrating fomepizole, specific enzyme inhibitor, or by giving ethanol, which has higher affin- ity to alcohol dehydrogenase, and competitively slows ethylene glycol metabolism (Patocka \& Hon, 2010). At this point (usually before renal failure develops) hemodialysis is indicated to clear ethylene glycol and its metabolites from plasma and, importantly, to effectively fight metabolic acidosis and should be continued until ethylene glycol levels are undetectable. Subsequent dialysis sessions are usually performed in the course of kidney failure as renal replacement therapy and continued until renal recovery. In most cases renal recovery is complete with restoration of normal kidney function.

\section{CASE PRESENTATION}

A fifty-two years old farmer with a history of unsystematically treated hypertension presented to the emergency department of the local hospital because of feeling unwell over the last couple of days, anuria lasting for 2 days and progressive edema. He was diagnosed with an advanced renal failure (serum creatinine $13.4 \mathrm{mg} / \mathrm{dL}$, BUN $161 \mathrm{mg} / \mathrm{dL}$ ), and partially compensated metabolic acidosis ( $\mathrm{pH} 7.33$, bicarbonate $13.8 \mathrm{mmol} / \mathrm{L}$, base excess $-12.1 \mathrm{mmol} / \mathrm{L})$. On admission he also had a moderate hyponatremia of $128 \mathrm{mmol} / \mathrm{L}$ and hyperkalemia of 5.5 $\mathrm{mmol} / \mathrm{L}$. Slight anemia was present with a hemoglobin level of $12.6 \mathrm{~g} / \mathrm{dl}$. Urinalysis revealed specific gravity of 1015 , and heavy proteinuria $(2150 \mathrm{mg} / \mathrm{dl})$, with normal sediment. Liver function tests were normal. On the ultrasound scan both kidneys were enlarged (measuring 13.8 and $14.6 \mathrm{~cm}$ ), hyperechogenic with normal cortex appearance and swollen pyramids. Chest X-ray was unremarkable. There was no history of recent infection or drug treatment.

Dialysis catheter was inserted into the right subclavian vein; patient underwent 2 dialysis sessions and was transferred to the Department of Nephrology for diagnostic workup.

On admission he was fully alert, well nourished, with no major complaints and physical examination was unremarkable apart from a central line in the right subclavian. Despite no drug history he was given $30 \mathrm{mg}$ of prednisone in suspicion of presumable acute interstitial nephritis, but remained anuric. Rabdomyolysis was excluded as a cause of renal injury. Anti Neutrophil Cytoplasm Antibodies (ANCA), Anti Nuclear Antibodies (ANA), anti Glomerular Basement Membrane (anti-GBM) anti-

e-mail: tlib@gumed.edu.pl

Abbreviations: ANCA, anti neutrophil cytoplasm antibodies; ANA anti nuclear antibodies; nti-GBM, anti-glomerular basement membrane. 


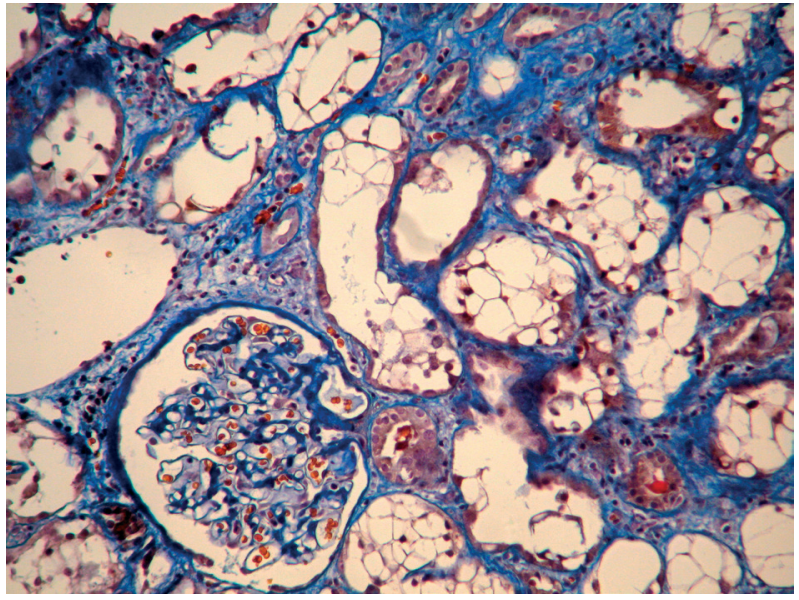

Figure 1. Kidney biopsy, AFOG staining, magnification 200x. Acute injury of tubular epithelium. Visible vacuolisation and baloon-like distention of a cytoplasm in most tubular cells. Small foci of interstitial inflammation, interstitial fibrosis and tubular atrophy. Normal glomerulus.

bodies were negative and complement C3 and C4 levels were normal. As no apparent cause of renal injury was found and there was no improvement, on the $15^{\text {th }}$ day of anuria renal biopsy was performed. It revealed signs of an acute tubular injury with diffuse vacuolization and balloon-like distention of the cytoplasm of mainly proximal tubules. There was mild inflammatory, predominantly mononuclear, infiltration in the interstitium. Calcium oxalate crystals were seen in many tubular lumens (Figs. 1 and 2). The glomeruli appeared normal. This pattern was regarded pathognomonic for injury related to ethylene glycol toxicity. On further enquiry patient admitted that 9 days before hospital admission, while repairing radiator in his car, he had aspirated antifreeze into his mouth several times but had spitted it out. He knew that antifreeze was toxic but did not regard this incident as serious. He had headache and nausea that evening and drunk about $250 \mathrm{ml}$ of $40 \%$ alcohol.

In the subsequent course patient was treated with maintenance hemodialysis. Diuresis gradually increased and his kidney function recovered. Dialysis was stopped after 3.5 months. He is now followed in nephrology outpatient department on antihypertensive medication, with serum creatinine level of $1.4 \mathrm{mg} / \mathrm{dl}$.

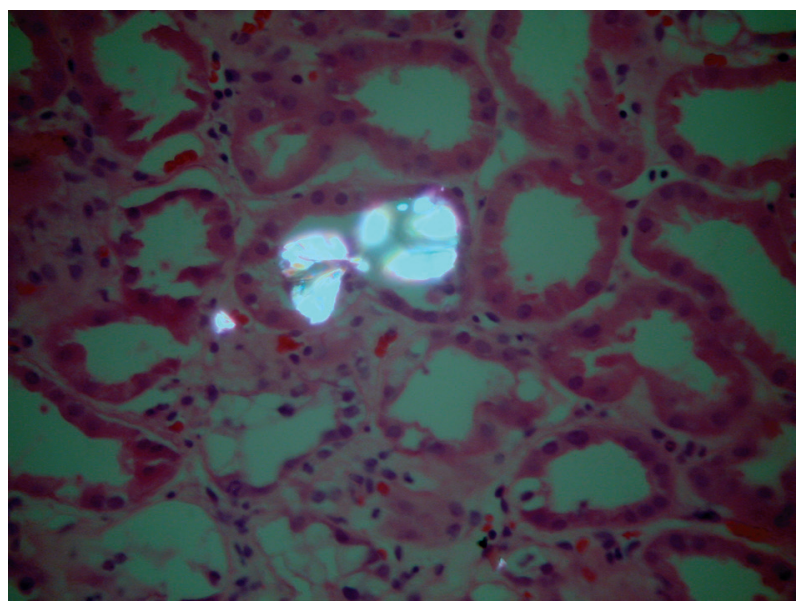

Figure 2. Kidney biopsy, H-E staining, magnification 200x

Acute injury of tubular epithelium, mononuclear inflammatory infiltrate in the interstitium. Calcium oxalate crystals visible in polarized light inside the tubular lumen.

\section{DISCUSSION}

Ethylene glycol intoxication is a relatively common cause of an acute renal injury. The most common source of ethylene glycol are antifreeze fluids used for car cooling systems, but it can also by found in the air conditioning systems or windshield deicers. It is colorless and odorless and has a sweet taste. Although intoxication through injured skin has been reported, most poisonings happen due to the oral ingestion. They are usually unintentional, however, ethylene glycol can be consumed as a cheap alcohol substitute or for intended suicide. In 2005, more than 6000 exposures to ethylene glycol were recorded in US with most cases being unintentional poisoning, not related to occupation (Lai et al., 2006). In Poland during years 1980-2000 it was responsible for $2.0-0.5 \%$ of acute poisonings referred to toxicology centers, with a downward trend (Kotwica \& Czerczak, 2007). However, the reported mortality in these patients was relatively high at about $30 \%$, being the second highest among all reported toxin groups. Only mortality related to methanol intoxication was higher than the ethylene glycol.

The clinical picture of ethylene glycol intoxication in our patient did not follow the typical course, as there was no significant neurodepressive phase usually seen even in mild intoxication. In retrospection, patient recalled only nausea, which could be attributed to acute poisoning. However, 7 days after ethylene glycol ingestion he developed toxic kidney injury as manifested by anuria, and presented to the hospital 2 days later, with already established renal failure requiring immediate dialysis. Importantly, although he knew that he had had contact with a toxic substance, he did not consider it important and relevant to his illness. Since the clinical course was not typical for ethylene glycol intoxication, he was not directly questioned about possible toxic ingestion. The microscopic findings in the renal tissue were crucial for the disease recognition, which strongly undermines the importance of kidney biopsy in the setting of unexplained acute kidney injury. The atypical course of ethylene glycol intoxication with the absence of an acute neurodepressive phase was probably due to a low amount of the ingested toxin and, more importantly, due to concomitant consumption of alcohol. Presumably, ethanol which is a strong competitive inhibitor of alcohol dehydrogenase, slowed down the metabolism of ethylene glycol and the appearance rate of its toxic metabolites: glycoaldehyde, glycolic acid and glioxalyc acid, thus reducing metabolic acidosis and related clinical deterioration. Nevertheless, oxalic acid, the end-product of the ethylene glycol metabolism accumulated and, by forming calcium oxalate crystals, caused organ injury.

Only several case presentations describe similar course of ethylene glycol intoxication, where patients presented late, with established organ damage: renal failure or severe peripheral neuropathy (Kaiser et al., 1993; Baldwin \& Sran, 2010). In all cases diagnosis of poisoning and identification of toxin was established only after extensive case analysis and investigations.

\section{REFERENCES}

Baldwin F, Sran H (2010) Delayed ethylene glycol poisoning presenting with abdominal pain and multiple cranial and peripheral neuropathies: a case report. I Med Case Reports 4: 220.

Kaiser W, Steinmauer H-G, Biesenbach G, Janko O, Zazgornik J (1993) Chronic ethylene glycol intoxication. Dtsch med Wochenschr 118: $622-626$ 
Kotwica M, Czerczak S (2007) Acute poisonings registered since 1970: trends and characteristics. Analysis of the files collected in the National Poison Information Centre, Łódź, Poland. Int J Occupat Med Environ Health 20: 38-43.

Kraut JA, Kurtz I (2008) Toxic alcohol ingestions: clinical features, diagnosis, and management. Clin J Am Soc Nephrol 3: 208-225.

Lai MW, Klein-Schwartz W, Rodgers GC et al. (2006) Annual report of the American Association of poison control centers' national poisoning and exposure database. Clin Toxicol 44: 803-932.
Patocka J, Hon Z (2010) Ethylene glycol, hazardous substance in the household. Acta Medica (Hradec Králové) 53: 19-23.

Reddy NJ, Suriawinata AA, Sedlacek M (2006) The importance of recognizing whewellite. Nephrol Dial Transplant 21: 2667. 